\title{
The Respiratory Therapy Practice-Based Outcomes Initiative (RT-PBOI): Developing a framework to explore the value added by respiratory therapists to health care in Alberta
}

\author{
Roberta Dubois RRT MA FCSRT ${ }^{1}$, Rena Sorensen RRT MSc FCSRT CAE ${ }^{2}$, Bryan Buell RRT BGS CTAJ ${ }^{3}$, \\ Tracey Telenko RRT BTech FCSRT ${ }^{4}$, Andrew West EdD FCSRT ${ }^{5}$
}

\begin{abstract}
R Dubois, R Sorensen, B Buell, T Telenko, A West. The Respiratory Therapy Practice-Based Outcomes Initiative (RT-PBOI): Developing a framework to explore the value added by respiratory therapists to healthcare in Alberta. Can J Respir Ther 2021;57:99-104. doi: 10.29390/ cjrt-2021-010.

Background: There exists a political imperative to have access to data that meets the needs of health care administrators, governments, and funding bodies to support evidence-informed decision making. It is incumbent upon respiratory therapists to examine how they can deliver the highest-quality patient care, but also that they add value to health systems that ensure the benefits of health innovations are shared equitably among all members of our communities.

Purpose: To explore the perceived value contributed by the respiratory therapy profession to health care and the health care system in the Province of Alberta at patient, team, and system levels.

Research methods: An interpretive descriptive approach was adopted, including the formation of a description and exploration of possible associations, relationships, and patterns within a field of practice.

Conclusions: The qualitative data analysis uncovered a framework that could inform research efforts of the respiratory therapy community in a way that contributes to the proposed mechanisms by which the profession generates value for the organization and patients. The RT-PBOI Conceptual Model identified five key concepts relating to the value contributed by respiratory therapists to health care: technical skills, practice across settings, strategic expertise, tools that leverage capacity, and growing value into the future.
\end{abstract}

Key Words: respiratory therapy; professional practice; practice patterns; research priorities

\section{INTRODUCTION}

There is a growing need for knowledge that can inform practice in the profession of respiratory therapy. Not only can this knowledge serve to inform practice trends, there also exists a political imperative to have access to data that meet the needs of health care administrators, governments, and funding bodies to support evidence-informed decision making.

With respect to the practice of respiratory therapists (RTs), it has been understood for some time that there are significant gaps between the best available evidence and what is often applied in clinical practice [1]. It is also recognized that initiating research in the profession can drive inquiry through difficult questions, and that quality improvement is an effective approach to challenging the status quo [2]. It is incumbent upon RTs to examine how they can deliver the highest quality patient care, but also that they add value to health systems that ensure the benefits of health innovations are shared equitably among all members of our communities [2]. The purpose of this qualitative investigation is to explore the perceived value contributed by respiratory therapy profession to healthcare and the health care system in the Province of Alberta at patient, team, and system levels.
The literature has shown that respiratory therapy practice is not compatible with unweighted metrics to determine optimal staffing levels [3]. For example, high-acuity patients have greater impact on RT workload such as a protective lung strategies on an unstable acute respiratory distress syndrome (ARDS) patient compared with a routine post-operative intensive care unit (ICU) stay. A relative value unit is required so that clinicians can flex patient care focus depending on the service need. The outcome of this work is an objective way to measure expected workload when indexed to fluctuations in patient acuity and volume. A respiratory index to guide staffing priorities is currently under development.

\section{BACKGROUND}

There is evidence to suggest that RTs are most valuable when their role focuses on the use of specialized expertise/skills to make autonomous decisions while providing protocol-driven care [4-8]. Protocols that allow RTs to practice to the full extent of their competencies, knowledge, and skills result in eliminating waste both in resources and wages [9] (compared with physicians), reduction in health service utilization and length of stay, and ventilator days [6]. The benefits are even more enhanced

${ }^{1}$ Provincial Respiratory Therapy, Alberta Health Services, Red Deer, Alberta, Canada; ${ }^{2}$ Central Zone Allied Health, Alberta Health Services, Ponoka, Alberta, Canada; ${ }^{3}$ College and Association of Respiratory Therapists of Alberta, Calgary, Alberta, Canada; ${ }^{4}$ Royal Alexandra Hospital, Alberta Health Services, Edmonton, Alberta, Canada; ${ }^{5}$ Canadian Society of Respiratory Therapists, Ottawa, Ontario, Canada

Correspondence: Rena Sorensen, Alberta Health Services, Courier: 307 a, 3rd Floor, 510249 Ave Ponoka, AB T4J 1R8, Mail: Ponoka Provincial Building Box 1000 Ponoka AB T4J 1R8. Tel: 403704 2564, Cell: 403963 7120, Fax: 403704 2581, E-mail: rena.sorensen@albertahealthservices.ca 
when costly interventions with a high volume of patients or protocols that enable RTs to practice at the top of their scope of practice are targeted $[6,9,10]$.

The existing traditional model of care where the RT is consulted by a physician provides significant value through improved patient outcomes when the RT receives ongoing training specific to evidence-based guidelines, pathways, and protocols $[5,7,10,11]$.

Adoption of evidence-informed practices is well recognized as an important factor in the provision of safe and effective care. De-Adoption is a crucial component of expanding practice in systems dealing with financial constraints. The De-adoption Conceptual Framework [12] supports the identification of low-value practices by prioritizing harm causation over lack of efficacy or cost effectiveness. The facilitation of de-adoption should include extensive education, relationship building, and the exploration of nonevidence factors like patient characteristics, perceived benefit/risk, feasibility, and clinician confidence [13]. Adequate resources need to be in place during de-adoption to both evaluate and sustain the effort. A summary of common critical care practices that RTs should consider for adoption and de-adoption are summarized in Table 1.

Generally, before a de-adoption initiative is considered, stakeholders need to see that the activities will redirect resources in an efficient way, and a differentiation between levels of interventions is recognized throughout (individual, policy, organization, payment) [14]. For example motivation to de-adopt an established practice will be more successful if the mechanism for change seems reasonable, there is a sense of urgency to act now, and it is easy to connect how the targeted changes interact with current practice activities [15].

In tandem with the need for evidence-informed practice, an imperative for the profession of respiratory therapy exists with respect to establishment outcome measures across health care systems. Health reform for respiratory therapy needs to include weighted standardized measures that incorporate cost savings over the entire episode of care [9], surveillance of health care utilization across the continuum of care, and a broad consideration of what resource utilization includes (equipment, medications, salary) [17]. It is also well established that one of the most important factors to incorporate in the generation of outcome measures and performance indicators are the perspectives of patients and their families [14].

In recognition of the need for outcomes for the profession, the Respiratory Therapy Practice-Based Outcomes Initiative (RT-PBOI) was implemented to explore, measure, evaluate, articulate, and foster the value that RTs contribute to the provision of evidence-informed respiratory care. Partnering RT-PBOI organizations (Alberta Health Services, College of Respiratory Therapists of Alberta, Canadian Society of Respiratory Therapists) are collaboratively seeking to examine the value that RTs contribute to health care teams in the provision respiratory care in a manner that addresses the challenge of translating this knowledge into action at the bedside and at the legislative and/or administrative table.

\section{METHODOLOGY}

This quality improvement initiative utilized a qualitative methodology to understand the perspectives of leaders in the respiratory therapy community from across Alberta Health Services regarding the values added by RTs to contribute to health care in Alberta. Specifically, the investigators sought to gain an understanding of the perspective of respiratory leaders to develop a framework to guide prioritization and implementation of future projects utilizing patient-oriented outcome measures and performance indicators. The following overarching research question guided the inquiry: How do leaders in respiratory therapy community in Alberta perceive value is added by RTs to evidence-informed health care?

An interpretive descriptive approach to inquiry was adopted for this quality improvement initiative. This inductive method of qualitative research involves the formation of a description and explores possible associations, relationships, and patterns within a field of practice [18]. Interpretive description therefore is well suited to address research questions in the health care context, while creating knowledge that is relevant for advancing disciplinary knowledge in respiratory therapy.

It was determined in consultation with the University of Alberta, that health research approval was not required in accordance with the Tri-Council Policy Statement 2 [19]. However, focus group participants were informed that the information collected would be used to inform a published article, as well as for an Alberta Health Services quality improvement project.

\section{PARTICIPANTS}

The College \& Association of Respiratory Therapists of Alberta (CARTA) assisted with the recruitment of 36 Respiratory Leaders from within the private, public, operational, and practice leadership perspective. All participants were registered respiratory therapists (RRTs) regulated members in good standing with CARTA. Participants included RRTs identified as leaders by CARTA and who were in attendance at its annual province-wide leadership meeting in Calgary, Alberta, in October 2018. During the day-long CARTA leadership meeting, participants were invited to a one hour focus group session. Not one participant in attendance opted to drop out of the focus group.

\section{DATA COLLECTION AND ANALYSIS}

The focus group session was organized using the modified open space technique. Six focus group facilitators (including four of the researchers and two volunteers with qualitative research experience) guided discussions. The six facilitators each undertook data collection during the event.

Participants self-selected into six groups (representing one of six "stations") initially for the first round of focus group discussions. There was a total of six stations, each station focusing discussion on one of three focus group questions:

\section{TABLE 1}

\section{Shared practices that impact the role of the respiratory therapist}

\begin{tabular}{ll}
\hline Adopt & De-adopt \\
\hline $\begin{array}{l}\text { Long-term disease prevention initiatives [9] } \\
\text { IV \& Central line Insertion [9] }\end{array}$ & High dose sedatives for ventilated patients [16] \\
$\begin{array}{l}\text { Ventilator management (early lung protective strategies, weaning, airway } \\
\quad \text { management, respiratory culture procurement, pneumonia prevention, high }\end{array}$ & $-\quad$ High PEEP \\
$\quad$ Inhaled nitric oxide \\
$\begin{array}{l}\text { Complex/chronic respiratory disease management, especially COPD [9,10] } \\
\text { Intubation [10] }\end{array}$ & $\begin{array}{c}\text { Secretion management strategies that include Incentive Spirometry [9], } \\
\text { Bronchoscopy \& BAL [10] }\end{array}$ \\
$\begin{array}{l}\text { NIV: (Pulmonary Edema, post-extubation, postop abdominal surgery when } \\
\quad \text { hypoxemic, COPDA/E) [10,12] }\end{array}$ & Withholding bronchodilator use in COPD because FEV1 does not change [16] \\
$\begin{array}{l}\text { Prone positioning [13] } \\
\text { IV Corticosteriod administration for COPD/Asthma acute exacerbations [16] }\end{array}$ & Applying oxygen to MI patients [16]
\end{tabular}

Note: $A R D S=$ Adult Respiratory Distress Syndrome; PEEP = Positive End Expiratory Pressure; COPD = Chronic Obstructive Pulmonary Disease;

$B A L=$ Bronchial Alveolar Lavage; FEV1 = Forced Expiratory Volume at 1 second; NIV = Non-Invasive Ventilation; COPDA/E = Acute Exacerbation of COPD,

$M I=$ Myocardial Infarction. 
- What respiratory therapy practices do you feel make the most valuable contributions to health care in Alberta?

- How can we enhance our capacity to excel as a profession?

- What do you consider to be the most important and/or relevant outcomes or impact of respiratory therapy interventions in the Alberta context?

Participants had an opportunity to contribute openly to the discussion, to share their perceptions openly with the group, or to privately share with the facilitator in writing using note pads. The full data set analyzed included transcribed focus group notes representing participant perceptions expressed for each of the three questions (collectively written on a shared paper/easel pad and on individual note pads), collected during 6 distinct focus group, as well as the field notes of researchers facilitating each focus group.

To remain open to unexpected findings, codes in this study were generated from immersion in the data. Building on a general understanding on the data, emergent codes were iteratively refined throughout the analysis. Figure 1 presents an overview of the analysis process.

During the first phase of data analysis, emergent first-round codes representing participant perceptions were inductively derived by all researchers on the team throughout this analysis. An initial exploratory analysis occurred throughout data collection, during focus group transcription, and then to the data as a whole, to support deep immersion in, and familiarization with, participant perceptions. As part of this process, researchers who fulfilled the roles of focus group facilitators reported initial perceptions of themes noted in their field notes and emanating during transcription focus group notes. Through discussion amongst the research team, these perceptions were consolidated into first-round codes.

During the second phase of data analysis, first-round codes were then applied to a second-round analysis of all data sets to produce rich information about the case relative to each theme for subsequent interpretation and reporting. Through a process of grouping similar codes undertaken by the Primary Investigator, an organizing system of five more concise themes were identified. The data analysis process was supported by the use of a qualitative software package (NVivo, Version 12, QRS international) [20].

\section{TRUSTWORTHINESS}

To ensure rigorous data collection and analysis into this study design, criteria for evaluating trustworthiness (credibility, dependability, and transferability) that parallel traditional approaches to determining validity (internal validity, external validity, and reliability) were employed [21].
To ensure credibility, corroborating evidence triangulated from six different focus groups (36 people) using multiple data sources (transcriptions of collected focus group responses, participant handwritten notes, and researcher field notes). Furthermore, focus groups were facilitated by six different researchers helping to ensure openness to divergent perspectives. In an effort to help identify and mitigate researcher biases, which are inherently rooted in preconceived positions, findings were shared during this iterative analysis process between members of the research team to identify alternate explanations.

Dependability refers to provision of a clear account of how data were collected and analyzed. Recognizing that the chosen method of data analysis may have substantial and unavoidable impact on the objects of inquiry, this study report includes a comprehensive account of how preliminary coding systems evolved into more clearly defined ones and then further into themes.

With respect to transferability, the ability of the reader to determine applicability to other practice and research settings, detailed contextual information and thick description has been used here. This approach heightens the ability of the reader to determine the potential for applying emergent understandings in another setting.

\section{RESULTS}

Emergent codes emanating from first round descriptive analysis of focus group leaders reported initial perceptions were corroborated across data sets. Through research team discussions, five primary themes emerged from a secondary analysis process of returning to reviewed literature and grouping similar first round codes: technical skills, practice across settings, strategic expertise, tools that leverage capacity, and the need to grow value into the future. Figure 2 provides a conceptual model of the relationships between these themes.

\section{TECHNICAL SKILLS}

RTs have skills and abilities that uniquely contribute to care in highly complicated, sometimes critical situations

- Focus on care of complex/higher-risk clientele

- Technical knowledge

- Ventilation and airway management

\section{PRACTICE ACROSS SETTINGS}

RTs employ approaches to practice that foster effective care in any health care setting, spanning across the continuum of care.

\section{FIGURE 1}

Data analysis process.

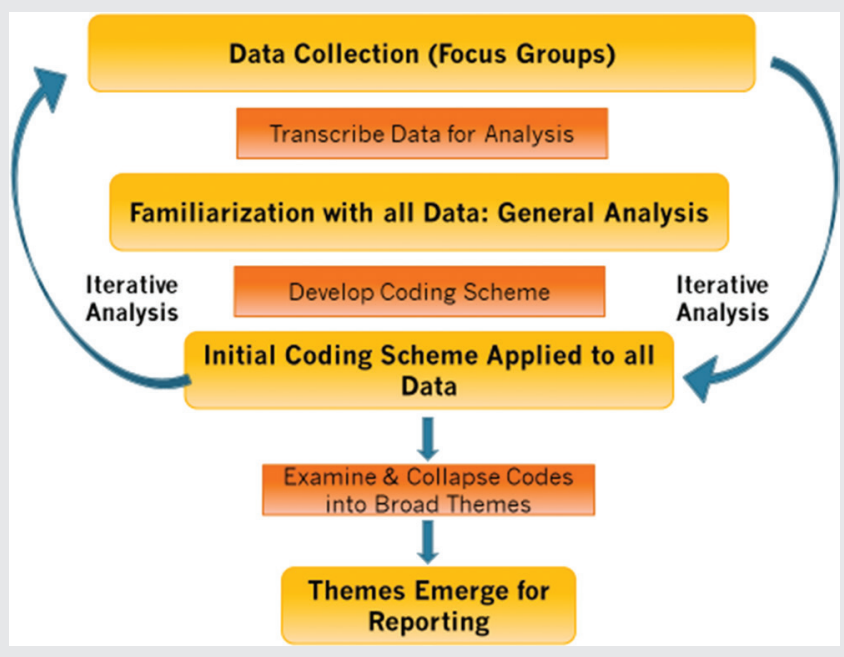


- Critical thinking

- Systems thinking capacity

- Critical decision-making

- Collaborative team-based care

- Case management and care planning

\section{STRATEGIC EXPERTISE}

RTs make a particularly impactful contribution to care in a number of priority areas of strategic importance

- Self-management programs

- Pulmonary rehabilitation

- Weaning protocols

- Respiratory disease pathways or order-sets

\section{TOOLS THAT LEVERAGE CAPACITY}

There exist important tools that help leverage RTs' skills and abilities to impact care and add value

- Formalized programs/protocols (Asthma Education Clinics, Pulmonary Rehabilitation, Respiratory Disease pathways/order sets, Self-Management Programs and weaning protocols)

- Leadership and engagement

- Research capacity

- Advancing the profession

\section{GROWING VALUE INTO THE FUTURE}

Growing the value added by RTs into the future will be supported and propagated by:

- Focused research need

- Establishing career pathways

This research endeavors to create a framework for a research strategy and identify areas of research that will define and enhance the value the profession is able to generate.

\section{DISCUSSION}

The qualitative data analysis uncovered a framework that could inform research efforts of the respiratory therapy community in a way that contributes to the proposed mechanisms (Figure 2) by which the profession generates value for the organization and patients. The RT-POI Conceptual Model defines value very broadly to include: clinical outcomes across the continuum of care, cost effectiveness, and patient satisfaction. The research team postulates that by establishing research across the profession that responds to the elements of the framework, operational leaders will have the evidence and information they need to satisfy healthcare administrators, governments, and funding bodies.

It is important to publish and disseminate the results of this quality improvement project to influence current and future research priorities within the respiratory therapy community. While the evidence is sparse, it remains important to acknowledge the quality improvement and research priorities that currently exist. There is something to be said about the practice issues that RTs are forced to confront. It is the opinion of the research group that the profession is constantly forced to prioritize quality improvement projects with the following characteristics:

- significant controversy and polarization

- low-frequency, high-risk competencies

- high cost or high mortality outcomes

- significant practice variations across a relatively small region

For instance, when the practice issue involves complex respiratory technology, there is simply no one else with the technical expertise to answer the question; thus, the respiratory community finds itself preoccupied with building knowledge and solutions around the issues described above.

The benefits of shaping respiratory therapy practice using the RT-PBOI conceptual model may provide some unforeseen benefits to the profession itself. Many facets within the model favor that RTs practice at the top of their scope [22], focus on the complex and high-risk patients, and are free to utilize critical thinking skills if supported with specialized training and evidence-informed protocols/programs. Understanding how the RT-BPOI Conceptual model broadens the perspective of the profession will enable a more inter-professional approach to practice. All of these characteristics have been linked to enhancing job satisfaction and staff retention [9].

The RT-PBOI will be fundamental the implementation of a series of unique knowledge translation projects that collectively contribute to achievement of the initiative's goal. Each individual project may represent one iteration (or portion of an iteration) of a respiratory therapy

\section{FIGURE 2}

RT-PBOI conceptual model. A conceptual model of the relationship between factors underlying the value contributed by respiratory therapists to healthcare (technical skills, practice across settings, strategic expertise, tools that leverage capacity). The ellipse representing the need to grow the value into the future touches on all four of these factors, representing their collective importance.

RT-PBOI = Respiratory Therapy Practice-Based Outcomes Initiative.

\section{Results}

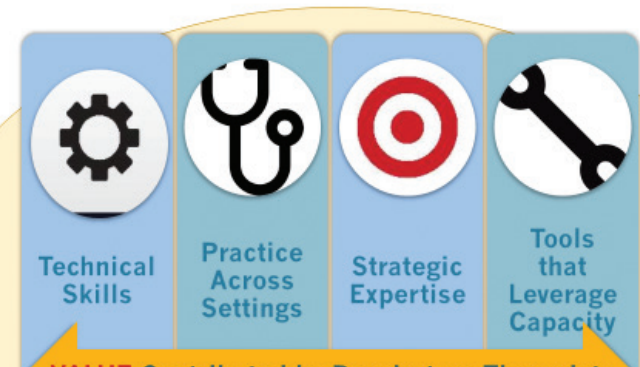

VALUE Contributed by Respiratory Therapists

Growing Value Into the Future 


\section{FIGURE 3}

RT practice-based knowledge to action process (Adapted from Graham et al. [23]).

$R T=$ Respiratory Therapy.

\section{RT Practice-Based Knowledge to Action Process}

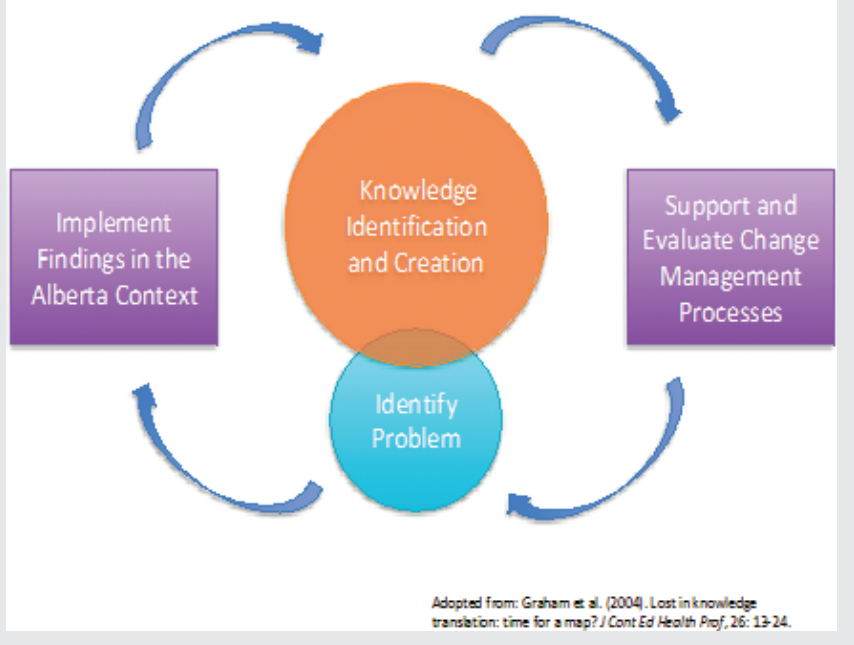

practice-based knowledge to action process (see Figure 3) and will serve to inform and guide future projects. The process adopted by this initiative will ensure that a meaningful body of knowledge is constructed, while enhancements to RT practice are accomplished.

\section{CONCLUSION}

There exist substantial gaps in literature with respect to the impact of Respiratory Therapy practice on patient outcomes. At the same time, there has been an emerging need to inform the advancement of respiratory profession by providing practice-oriented research that describes the impact and effectiveness of respiratory therapy on the health care systems and the health of patients. This quality improvement project sought to inform development of a research strategy framework to guide future outcomes-oriented inquiry, and to identify areas of research that will define and enhance the value the profession is able to generate.

This project identified five key concepts relating to the value contributed by RTs to health care: technical skills, practice across settings, strategic expertise, tools that leverage capacity, and growing value into the future. To adequately understand respiratory therapy practice these concepts can be examined using a knowledge to action approach that will enhance RTs' practice while building knowledge that describes the impact of that practice.

\section{Contributors}

\section{AUTHOR DISCLOSURES}

All authors contributed to the conception or design of the work, the acquisition, analysis, or interpretation of the data. All authors were involved in drafting and commenting on the paper and have approved the final version.

\section{Funding}

This study did not receive any specific grant from funding agencies in the public, commercial, or not-for-profit sectors.

\section{Competing interests}

All authors have completed the ICMJE uniform disclosure form at www. icmje.org/coi_disclosure.pdf and declare: no financial relationships with any organizations that might have an interest in the submitted work in the previous 3 years; no other relationships or activities that could appear to have influenced the submitted work.

\section{Ethical approval}

It was determined in consultation with the University of Alberta, that health research approval was not required in accordance with the Tri-Council Policy Statement 2. However, focus group participants were informed that the information collected would be used to inform a published article, as well as for an Alberta Health Services quality improvement project.

\section{REFERENCES}

1. Restrepo, RD. AARC clinical practice guidelines: from "reference-based" to "evidence-based." Respir Care 2010;55:787-9.

2. Nickerson JW. Message from the editor-in-chief. CJRT 2016. 52(4):103. doi: 10.1016/j.ipm.2015.12.015

3. AARC Position Paper: best practices in respiratory care productivity and staffing. 2012.

4. Becker EA, Hoerr CA, Wiles KS, Skees DL, Miller CH, Laher DS. Utilizing respiratory therapists to reduce costs of care. Respir Care 2018;63(1):103-11. doi: 10.4187/respcare.05808

5. Brilli R, Spevetz A, Branson R, Campbell G, Cohen H, Dasta J. Critical care delivery in the intensive care unit: defining clinical roles and the best practice model. Crit Care Med 2001;29(10):2007-19. doi: 10.1097/00003246-200110000-00026

6. Hess D. What is evidence-based medicine and why should I care. Respir Care 2004;47(7):730-41.

7. Koch R. Therapist driven protocols: a look back and moving into the future. Crit Care Clin 2007;23(2):149-59. doi: 10.1016/j.ccc.2006.11.012

8. Stoller J. The effectiveness of respiratory care protocols. Respir Care 2004:47(7):761-5.

9. Stoller J, Mascha E, Kester L, Haney D. Randomized controlled trial of physician-directed versus respiratory therapy consult service-directed respiratory rare to adult non-ICU inpatients [Electronic version]. Am J of Respir Crit Care Med 1998;158(4):1068-75. doi: 10.1164/ ajrccm.158.4.9709076

10. Kollef M, Shapiro S, Clinkscale D, Cracchiolo L, Clayton D, Wilner R. The effects of respiratory therapist-initiated treatment protocols on patient outcomes and resource utilization [Electronic version]. Chest 2000;117(2):467-75. doi: 10.1378/chest.117.2.467

11. Ely E, Meade E, Haponik M, et al. Mechanical ventilator weaning protocols driven by nonphysician health-care professionals: evidence-based clinical practice guidelines. Chest 2001;120(6 Suppl.):454S-63S. doi: 10.1378/chest.120.6_suppl.454S

12. Niven DJ, McCormick J, Strus SE, et al. Reproducibility of clinical research in critical care: a scoping review. BMC Med 2018;16:26. doi: $10.1186 / \mathrm{s} 12916-018-1018-6$ 
13. MunshI L, Gershengorn HB, Fan E, et al. Adjuvants to mechanical ventilation for acute respiratory failure: adoption, de-adoption, and factors associated with selection. Annals ATS 2017;14(1):94-102. doi: 10.1513/ AnnalsATS.201606-438OC

14. Weiner J. Rosenquist R. Issues at the heart of advancing the de-adoption of low-value care. LDI Issue Briefs 2017;21(6):116-21.

15. Rubin BK, Haynes JM. Myths, misunderstandings, and dogma in respiratory care. Resp Care 2012;8(57):1314-24. doi: 10.4187/respcare.01816

16. Prasad V, Cifu A, Ioannidis JP. Reversals of established medical practices. JAMA 2012;307(1):37-8. doi: 10.1001/jama.2011.1960

17. AARC Compensation Calculator. Available at: https://my.aarc.org/ Security/Sign-In?returnUrl=https://www.aarc.org/resources/tools-software/aarc-respiratory-therapist-human-resource-study-2014/ respiratory-therapist-human-resource-study-downloads/

18. Thorne S, Kirkham SR, MacDonald-Emes J. Interpretive description: a noncategorical qualitative alternative for developing nursing knowledge.
Res Nurs Health 1997;20(2):169-77. doi: 10.1002/ (SICI) 1098-240X(199704)20:2\%3C169::AID-NUR9\%3E3.0.CO;2-I

19. The Tri-Council Policy Statement 2 (TCPS2) governing research ethics in Canada. Available at: https://ethics.gc.ca/eng/documents/ tcps2-2018-en-interactive-final.pdf

20. NVivo 12, QSR International Pty Ltd.

21. Lincoln YS, Guba EG. But is it rigorous? Trustworthiness and authenticity in naturalistic evaluation. Naturalistic Evaluation 1986;1986(30):7384. doi: $10.1002 /$ ev.1427

22. National Competency Framework for the Profession of Respiratory Therapy. 2016. The national alliance of respiratory therapy regulatory bodies. Available at: https://www.csrt.com/wp-content/uploads/EN_ Framework_2016_NARTRB_NCF_Part1.pdf

23. Graham ID, Logan J, Harrison MB, et al. Lost in knowledge translation: time for a map? J Contin Educ Health Prof 2006;26(1):13-24. doi: 10.1002/chp.47 